\section{Errichtungsausschuss formiert sich neu}

\section{Landespflegekammer Niedersachsen besetzt Vorstand nach}

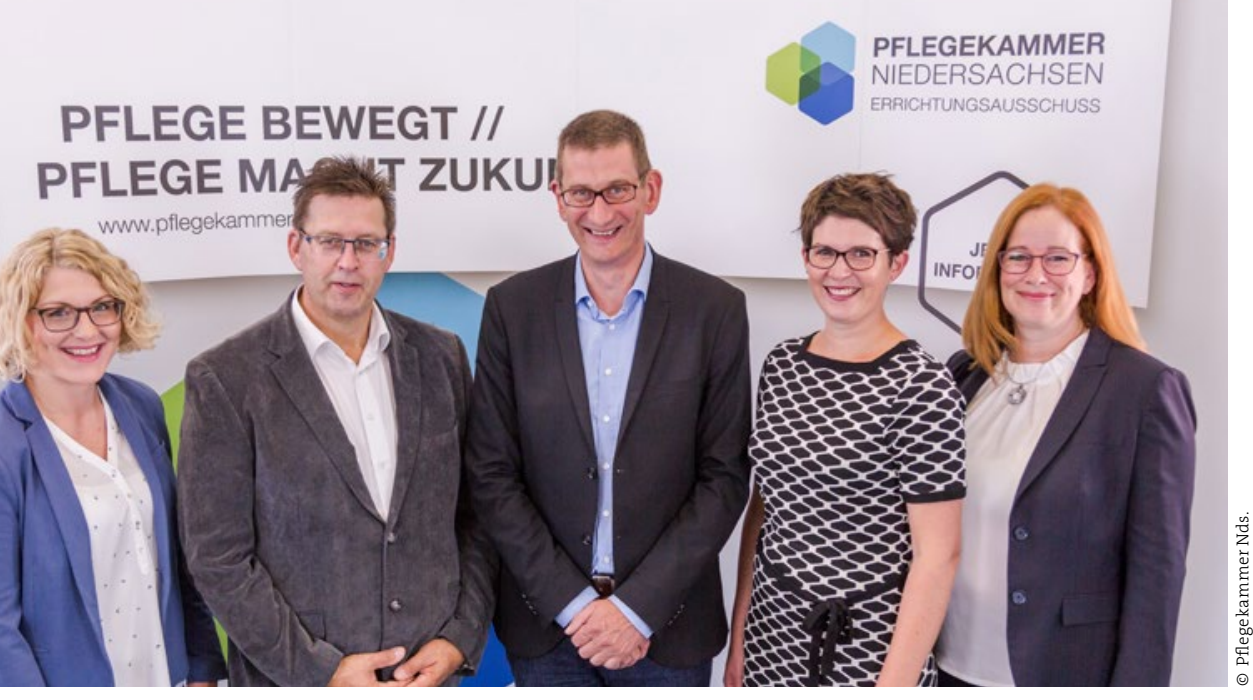

" Im Rahmen einer Sitzung des Errichtungsausschusses der Landespflegekammer Niedersachsen fand im August eine Nachbesetzung des Vorstandes statt.

Das neue Führungsteam besteht aus der Vorsitzenden des Errichtungsausschusses Katrin Havers (Gesundheitsund Kinderkrankenpflegerin), der stellvertretenden Vorsitzenden Sandra Mehmecke (Gesundheits- und Krankenpflegerin) und den Vorstandsmitgliedern Jürgen Butzke (Altenpfleger), Nadya Klarmann (Altenpflegerin) und Rolf Weiß (Gesundheits- und Krankenpfleger).

www.pflegekammer-nds.de

\title{
Bundespflegekammer kommt
}

\section{Meilenstein für gemeinsame und einheitliche Selbstverwaltung}

" Die Vorbereitungen für eine Bundespflegekammer haben begonnen. Am 15. August 2017 beschlossen der Deutsche Pflegerat (DPR) und die Landespflegekammer Rheinland-Pfalz die Errichtung einer Gründungskonferenz. Diese soll innerhalb von höchstens einem Jahr alle Vorbereitungen für eine Bundespflegekammer als Dach bestehender und künftiger Landespflegekammern treffen. Bisher hat die Landespflegekammer RheinlandPfalz ihre Arbeit aufgenommen, Initiativen für weitere Landespflegekammern gibt es in Schleswig-Holstein, Niedersachsen, Nordrhein-Westfalen und Baden-Württemberg. Die Bundespflegekammer soll eine ,gemeinsame und einheitliche Selbstverwaltung aller professionellen Pflegeberufe auf Bundesebene" sicherstellen.

www.deutscher-pflegerat.de

\section{Aufwertung der Sozial- und Gesundheitsberufe}

\section{BMFSFJ und BMAS starten gemeinsames Aktionsprogramm}

" Mehr gesellschaftliche Anerkennung und bessere Löhne in Sozial- und Gesundheitsberufen - das sind kurz zusammengefasst die erklärten Ziele des gemeinsamen Aktionsprogramms, das die Bundesministerin für Familie, Senioren, Frauen und Jugend, Dr. Katarina Barley, die Bundesministerin für Arbeit und Soziales, Andrea Nahles, und die Ministerpräsidentin des Landes Rheinland-Pfalz, Malu Dreyer, Ende August vorstellten.

Maßnahmen in den folgenden fünf Bereichen sollen dabei zum Ziel führen:
•• Eine kostenfreie und vergütete Ausbildung

- Bessere Löhne im Gesundheits- und Sozialwesen

-• Bessere und vielfältigere Entwicklungsperspektiven

- Stärkung der Arbeitszufriedenheit

- Die Vermeidung von Berufsausstiegen sowie die nachhaltige Sicherung und Weiterentwicklung der Fachkräftebasis

Bundesfamilienministerin Barley setzt darauf, die Attraktivität der Berufe zu erhöhen: „Das schaffen wir [...] durch bessere Arbeitsbedingungen, durch höhere Personalschlüssel und berufliche Entwicklungsmöglichkeiten," so die Ministerin.

Der Deutsche Pflegerat e. V. (DPR) begrüßte die Initiative und betonte, dass es wichtig sei, die Arbeitsbedingungen von Pflegenden zu verbessern. Dazu gehöre auch die ,zügige Festschreibung von Mindestpersonalvorgaben im Krankenhausbereich" und dass die "Stimme“ der Pflege gehört und ernstgenommen werde, forderte Andreas Westerfellhaus.

www.bmfsfj.de 